\title{
Metaplasia ósea endometrial Reporte de un caso
}

\author{
Pío Iván Gómez Sánchez*; Luis Humberto Beltrán CH.**; Lilia Sánchez***
}

RESUMEN: Se presenta un caso raro de metaplasia ósea endometrial en una paciente de 22 años de edad, quien consultó por amenorrea secundaria de tres años de evolución posterior a un legrado realizado por aborto incompleto.

Se realizó histeroscopia operatoria, dilatación y curetaje extrayendo fragmentos óseos cuyo estudio anatomopatológico confirma el diagnóstico de metaplasia ósea endometrial.

Se hace discusión del tema y se exponen las diferentes teorías sobre su patogénesis.

PALABRAS CLAVES: Metaplasia ósea endometrial, histeroscopia operatoria.

SUMMARY: We report a rare case of endometrial osseous metaplasia in a 22 years old patient with three years of secundary amenorrea after espontaneus abortion.

An endoscopic approach was performed obtained osseus fragments. The pathologic study confirmed endometrial osseous metaplasia.

In this article theories and pathogenesis of endometrial osseous metaplasia are reviewed.

KEY WORDS: Endometrial osseous metaplasia, operative hysteroscopy.

Se trata de una paciente de 22 años de edad, quien consultó por amenorrea de tres años de evolución, posterior a legrado uterino realizado por aborto incompleto espontáneo. No refería otra sintomatología. Había sido tratada con estrógenos conjugados y en otras ocasiones estrógenos más progestágenos sin que se consiguiera sangrado.

Como antecedentes de importancia: menarquia a los 14 años, G3P2V2A1, fecha de último embarazo Dic. 93.

El examen físico general se encontró dentro de límites normales, el examen ginecológico mostró genitales externos normales, especuloscopia normal y al tacto vaginal: útero en anteversoflexión con tamaño total de ocho centímetros, fondos de saco libres y anexos normales.

La ecografía transvaginal, mostró: útero de $80 \times 41 \times$ $75 \mathrm{~mm}$. y se observaba hiperrefringencia del eco endometrial. Ver Figura 1

Es remitida a la Unidad de Cirugía Laparoscópica del Instituto Materno Infantil de Santafé de Bogotá y con

\footnotetext{
* Profesor Asociado y Director. Departamento de Ginecología y Obstetricia. Universidad Nacional de Colombia. Instituto Materno Infantil.

** Ginecoobstetra. Universidad Nacional de Colombia. Instituto Materno Infantil.

Instructora Asociada. Departamento de Patología. Universidad
} Nacional de Colombia. Instituto Materno Infantil. sospecha diagnóstica de metaplasia ósea endometrial se realizó Video laparo-histeroscopia, en la que se evidencia formación ósea irregular con espículas que ocupa toda la cavidad endometrial. Ver Figura 2

Se practicó extracción histeroscópica de éstas formaciones. Durante el procedimiento se presentó en forma accidental perforación uterina fúndica por lo que se realizó histerorrafía con dos puntos intracorpóreos realizados mediante Videolaparoscopia, encontrándose adicionalmente adherencias perihepáticas.

El estudio histopatológico mostró metaplasia ósea con fragmentos de tejido fibroconectivo con inflamación aguda y crónica. Ver Figura 3

\section{Discusión}

Se han reportado alrededor de 50 casos en la literatura Mundial de esta entidad conocida como "osificación", "retención de hueso fetal", "metaplasia ósea" uterina o endometrial $(1,2)$.

La metaplasia hace referencia al reemplazo focal del estroma endometrial por un tejido mesenquimal no neoplásico, el cual habitualmente no se encuentra en el endometrio normal.

En raras ocasiones se han encontrado focos de tejido óseo o cartílago a nivel de la mucosa endometrial. Cuando esto se ha visto usualmente ha sido después de un aborto. En estas ocasiones podría tratarse de una verda- 
Figura 1

ECOGRAFIA TRANSVAGINAL

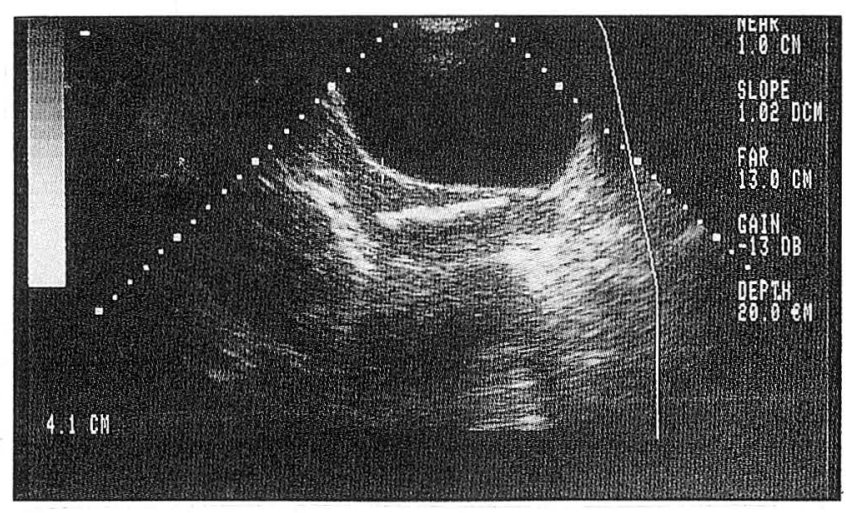

Se observa patrón hiperrefringente intrauterino

Figura 2

IMAGEN HISTEROSCOPICA DE FORMACION OSEA CORALIFORME

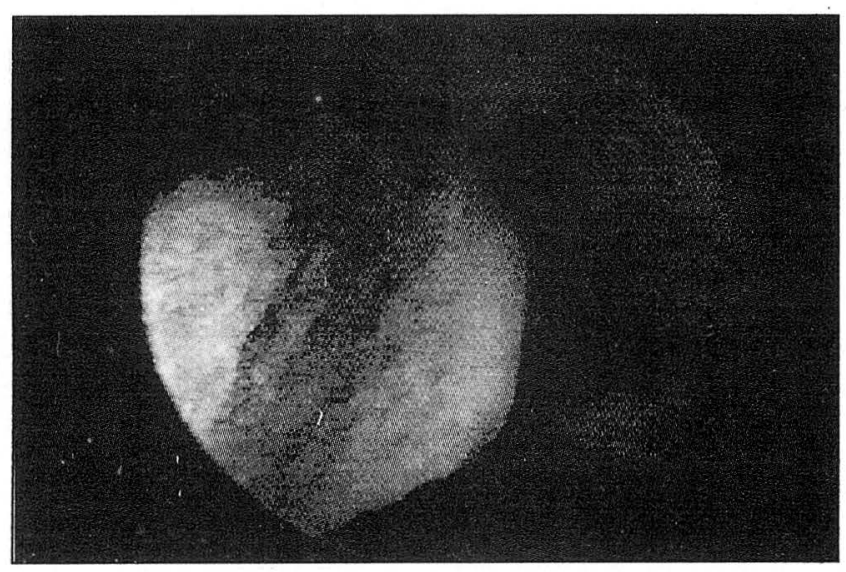

Figura 3

COLORACION H-E 100X ENDOMETRIO CON PRESENCIA DE ESPICULAS OSEAS, RODEADAS DE INFILTRADO LINFOPLASMOCITARIO

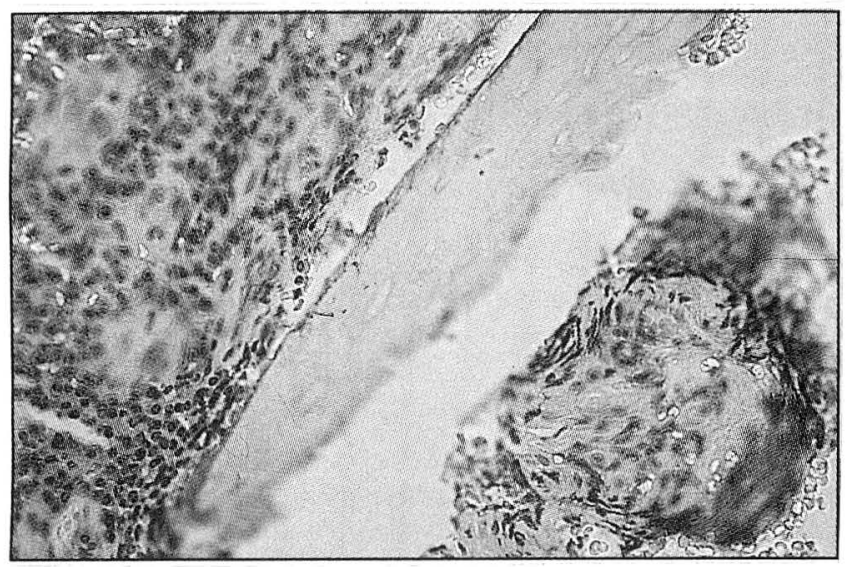

dera metaplasia que surge directamente a partir de las células estromales endometriales. Esta metaplasia puede ser también un hallazgo frecuente dentro del estroma de carcinomas o sarcomas endometriales.

La formación de hueso en el útero es una forma rara de heterotopía que implica la presencia de tejido maduro en una localización anormal.

Otras formaciones heterotópicas en el útero descritas, son: la presencia de cartílago, músculo liso y tejido glial (3-5).

El hallazgo de hueso heterotópico no es infrecuente en pacientes con historia de abortos repetidos y endometritis. En casos muy raros el hueso representa un fenómeno metaplásico verdadero ocasionado por una reacción inflamatoria. En muchos casos, la fuerte asociación con un embarazo previo, la inmadurez de los elementos heterólogos, y lo raro de la metaplasia ósea en otros tipos de endometritis representa una forma de implantación de partes fetales (8).

En este caso la mucosa endometrial muestra los cambios por una endometritis crónica activa y necrosis $\sin$ ninguna evidencia de actividad regenerativa, lo cual podría estar implicando un papel inhibitorio en el crecimiento tisular a partir del tejido óseo metaplásico. La necrosis endometrial está demostrada por la pérdida de la arquitectura tisular y un extenso infiltrado inflamatorio mixto con neutrófilos, linfocitos y plasmocitos. Las trabéculas óseas están desvitalizadas. El origen de estas trabéculas podría estar a partir de un embrión. La necrosis a partir de un tejido necrótico heterotópico es lo menos probable (6).

Ocasionalmente un aborto se ve complicado por el desarrollo de una osificación endometrial. Esta parece ser más la consecuencia de una inflamación crónica prolongada en un tejido débil y debe ser diferenciada de remanentes fetales, pero a pesar del aumento en los abortos terapéuticos, en países donde el aborto es legal, no se ha visto relacionado a su vez con el incremento de este problema. (7)

En la actualidad no se conoce en forma cierta la patogénesis de esta entidad. En 1884 Virchow atribuyó la formación de hueso en el endometrio a la diferenciación de fibroblastos en osteoclastos, posteriormente Brocq, propuso que la estimulación estrogénica continúa, podría ser el factor más importante, lo cual fue refutado por Hamand quien sostuvo que el endometrio osificado tenía pobre respuesta a las hormonas. El alto consumo de calcio y vitamina $\mathrm{D}$ fue propuesto, como mecanismo pero estudios posteriores lo negaron.

Houlne, propuso que la presencia de hueso fetal es un importante factor, pero ocurre formación ósea postaborto en etapas tan tempranas donde aún no hay formación de hueso fetal. Gerbié describe que la inflamación crónica prolongada es quizás el factor más importante (3,5-6). La inflamación asociada al proceso reparativo postaborto puede jugar un importante papel en la formación ósea heterotópica. La mayoría son pacientes en edad reproductiva y más comúnmente consultan por trastornos del ciclo menstrual dados por amenorrea, oligomenorrea y/o descarga vaginal irregular. La infertilidad es muy frecuente en éstas pacientes y se puede dar 
por varias razones. La presencia de tejido óseo en el endometrio puede actuar en forma semejante a un dispositivo intrauterino y se han descrito niveles de prostanoides (PgF2a), elevados en líquido endometrial, en estos casos la obstrucción del canal endometrial y/o de las trompas y la alteración del lecho de nidación son otros factores relacionados (10).

Aunque casi siempre han sido un hallazgo histológico, se han visto macroscópicamente en especímenes de biopsia, histerectomía o curetaje como espículas óseas múltiples que llegan a tener un tamaño de $2 \mathrm{~cm}$.

En algunas pacientes se ha encontrado este tipo de material incluso en dos o más especímenes en un período de hasta tres años. En el examen histológico se observan espículas óseas viables o necróticas maduras dentro del estroma endocervical, endometrial o miometrial. Puede haber una asociación con una endometritis crónica severa.

No se conoce un tratamiento definitivo, particularmente en mujeres jóvenes a quienes se les debe conservar su función reproductiva. Quizás una serie de dilataciones y curetajes para remover el tejido óseo es preferible a un curetaje único y vigoroso. El uso de la histerectomía electiva se debe limitar a mujeres con paridad satisfecha o para aquellos casos en los cuales los problemas menstruales incapacitantes no responden a las dilataciones y curetajes.

El uso de hormonas estrogénicas para acelerar la regeneración del endometrio es controversial ya que algunos reportes las incriminan como factor patogénico (3).

Actualmente la histeroscopia diagnóstica y terapéutica es el mejor abordaje, que permite en un mismo acto el enfoque integral. Es importante como se hizo en este caso, practicar la histeroscopia y laparoscopia simultáneamente, lo que disminuye el riesgo siempre presente de perforación uterina. Sin embargo a pesar de lo anterior se puede lesionar el útero, circunstancia que debe la paciente conocer con anterioridad, cuando el médico explica los riesgos del procedimiento en la firma del consentimiento informado (artículo 15, ley 23 de 1981)(12).

Cuando se presenta una lesión uterina, se debe terminar la evacuación del contenido uterino bajo visión directa laparoscópica y al finalizar se hará el tratamiento laparoscópico, colocando puntos seromusculares en el sitio de la lesión, anudando intra o extracorpóreamente.

\section{BIBLIOGRAFIA}

1. Clement, P.B., Tumorlike lesions of the uterine corpus in Tumors and tumorlike lesions of the uterine corpus and cervix. Edited by Clement, P. B., and Young Robert. Churchill Livingstone. 1993:150-151

2. Kaminski, P., Beningn conditions of the uterus. En: Clinical Gynecologic Pathology. Hernández, E., Atkinson,B.E. Ed: Saunders. 1996: $251-252$.

3. Bhatia, N., Hoshiko, M., Uterine Osseous Metaplasia. Obstet Gynecol 1982; 60: 256-259.

4. Longacre, T., Kempson, R. Endometrial Inflammation. En: Obstetrical and Gynaecologycal Pathology. Haines, P., Taylor, H.E. Churchill Livingstone. 1995; 409-410.

5. Silvergberg, S.G., Kurman, R.J., Tumors of the Uterine Corpus and Gestational Trhophoblastic Disease. Atlas of Tumor Pathology. AFIP. Third Series. Fascicle 3. Editor: Rosai, J. 1992; 207.

6. Hustin, J., Bulletin de la División Francais de l'AIP No. 23-junio 1996.
7. Buckley, C.H., Endometrial Inflamation. En: Haines and Taylor. Obstetrical and Gynaecologycal Pathology. Edited by Fox. Churchill Livingstone. 4th Edition. 1995; 406.

8. Kurman, R.J. Benign disease of the endometrium. En: Kurman, R.J. Pathology of the Female Genital Tract. Ed: Springer-Verlagi. 3a Ed. 1987; 310

9. Dawood, Y., Jarrett, J. Prolonged intrauterine retention of fetal bones after abortion causing infertility. Am J Obstet Gynecol. 1982; 113: 715-717.

10. Ganem, K. Endometrial ossification. Am J Obstet Gynecol. 1962; 83: 1592-1594.

11. Ceccacci, L., Clancy, G. Endometrial Ossification. Report of an additional case. Am J Obstet Gynecol. 1981; 141: 103-104.

12. Ley 23 de 1981. Código de Etica Médica. 